\title{
A Prediction Model for Falls in Community-Dwelling Older Adults in Podiatry Practices
}

\author{
Danique J.J. van Gulick ${ }^{a, b}$ Sander I.B. Perry ${ }^{b, c}$ Marike van der Leeden ${ }^{d, e}$ \\ Jolan G.M. van Beek ${ }^{a}$ Cees Lucas ${ }^{b, c}$ Martijn M. Stuiver ${ }^{b, c}$ \\ aDepartment of Science and Data-analysis, RondOm Podotherapeuten, Podiatric Primary Care Center, Leusden, \\ The Netherlands; bepartment of Epidemiology and Data Science, University Medical Center Amsterdam, University \\ of Amsterdam, Amsterdam, The Netherlands; ' Amsterdam Public Health Research Institute, University Medical \\ Center Amsterdam, Amsterdam, The Netherlands; ${ }^{d}$ Amsterdam Rehabilitation Research Centre, Reade, Amsterdam, \\ The Netherlands; 'Department of Rehabilitation Medicine, University Medical Center Amsterdam, Vrije Universiteit, \\ Amsterdam, The Netherlands
}

\section{Keywords}

Fall risk · Foot · Podiatrist - Assessment tool

\begin{abstract}
Introduction: Falls are a worldwide health problem among community-dwelling older adults. Emerging evidence suggests that foot problems increase the risk of falling, so the podiatrist may be crucial in detecting foot-related fall risk. However, there is no screening tool available which can be used in podiatry practice. The predictive value of existing tools is limited, and the implementation is poor. The development of risk models for specific clinical populations might increase the prediction accuracy and implementation. Therefore, the aim of this study was to develop and internally validate an easily applicable clinical prediction model (CPM) that can be used in podiatry practice to predict falls in community-dwelling older adults with foot (-related) problems. Methods: This was a prospective study including community-dwelling older adults ( $\geq 65$ years) visiting podiatry practices. General fall-risk variables, and foot-related and function-related variables were considered as predictors for the occurrence of falls during the 12-month follow-up. Logistic regression analysis was used for model building, and
\end{abstract}

karger@karger.com www.karger.com/ger

Karger"

GOPEN ACCESS
(C) 2022 The Author(s)

Published by S. Karger AG, Basel

This is an Open Access article licensed under the Creative Commons Attribution-NonCommercial-4.0 International License (CC BY-NC) (http://www.karger.com/Services/OpenAccessLicense), applicable to the online version of the article only. Usage and distribution for commercial purposes requires written permission. internal validation was done by bootstrap resampling. $\boldsymbol{R e}$ sults: 407 participants were analyzed; the event rate was $33.4 \%$. The final model included fall history in the previous year, unsteady while standing and walking, plantarflexor strength of the lesser toes, and gait speed. The area under the receiver operating characteristic curve was 0.71 (95\% Cl: 0.66-0.76) in the sample and estimated as 0.65 after shrinkage. Conclusion: A CPM based on fall history in the previous year, feeling unsteady while standing and walking, decreased plantarflexor strength of the lesser toes, and reduced gait speed has acceptable accuracy to predict falls in our sample of podiatry community-dwelling older adults and is easily applicable in this setting. The accuracy of the model in clinical practice should be demonstrated through external validation of the model in a next study.

(c) 2022 The Author(s).

Published by S. Karger AG, Basel

\section{Introduction}

Falls are an increasing health problem in the growing population of older adults. Every year, almost $33 \%$ of all community-dwelling older adults fall [1]. Falls can lead to injuries, morbidity, and mortality, and thereby consti- 
tute a major financial burden for health care [1]. In 2019, the total amount of direct medical costs in the Netherlands was EUR 1.09 billion [2].

Since there is emerging evidence about the increased risk of falling in community-dwelling older adults with foot problems, foot assessment should be a routine component in fall prevention [1,3-7]. The podiatrist is a healthcare provider diagnosing and treating foot problems, and is therefore ideally equipped to contribute to fall-risk screening [8].

To identify patients who are at risk of falling, clinical guidelines recommend every healthcare provider at least to ask about falls in the previous year in older adults $[1,3$, 9]. However, this single question is not sufficiently accurate to determine the risk of future falls [10]. In order to further support healthcare providers in fall risk assessment, many assessment tools, including questionnaires, physical performance tests, screening models, and algorithms, have been developed $[1,10,11]$. Despite the recommendations in clinical guidelines and available tools, in many areas of the world, fall risk assessment is currently scarcely implemented in clinical practice [12]. Unknown validity, a long administration time, insufficient availability of equipment in clinical (podiatry) practice, and unfamiliarity of the healthcare provider with the available tools are reasons for a low assessment rate [1, 12]. In addition, the predictive value of the available tools to distinguish between fallers and nonfallers in community-dwelling older adults remains limited $[1,10,11]$. This may be due to the failure to take into account the multifactorial cause of falling $[10,11]$, and methodological approaches in development studies, including the lack of tailoring to populations with specific characteristics [13].

The community-dwelling older adult population consulting the podiatrist is one such population with specific characteristics. Foot (-related) problems, diabetes, and their sequelae are the most prominent disorders in this population [8]. Despite the known increased risk of falling in these older adult population $[4,14]$, there is no easily applicable and accurate screening tool available which can be systematically used in podiatry practices to determine fall risk. Improving fall risk prediction accuracy in podiatry practice could support and stimulate podiatrists in identifying high-risk patients in clinical practice. After identifying a patient at higher risk, the podiatrist could make the patient aware, consider the need for referral to a relevant specialist or fall prevention program, or initiate appropriate evidence-based interventions [15]. Development of a clinical prediction model (CPM) tailored to the podiatry population by adding foot-related variables to general known fall-risk predictors such as fall history in the previous year might increase prediction accuracy. Therefore, the aim of this study was to develop and internally validate an easily applicable CPM tailored to the older adult podiatry population.

\section{Materials and Methods}

\section{Study Design and Participants}

We conducted a prospective study at RondOm Podotherapeuten, a podiatric primary care organization in the Netherlands, between October 2017 and February 2020. Community-dwelling older adults who planned a consultation with a podiatrist at one of the 134 practices of RondOm were consecutively recruited. We selected patients based on the following criteria: age $\geq 65$ years, living in the community, and indicated for a podiatric consultation. Patients who were unable to walk $6 \mathrm{~m}$, could not stand unassisted for $30 \mathrm{~s}$ without shoes, had insufficient command of Dutch or English, or had severe cognitive impairments precluding reliable (self-report) data collection at baseline or follow-up were excluded. The secretariat of RondOm who planned the patients consultation and the podiatrist who was consulted both checked the eligibility criteria. Patient characteristics (age, sex, length, weight, body mass index, medical conditions [diabetes, osteoarthritis, and central nervous system disease], and amputations in the foot/ankle region) were collected during the consultation by the podiatrist. Patients who participated in the study received usual podiatric care. All participants gave written informed consent. This study was waived from formal medical ethical evaluation by the Dutch Central Committee on Research Involving Human Subject (CCMO). Ethical standards adhered to the Declaration of Helsinki.

\section{Predictors}

Candidate predictors were selected based on best available evidence in the literature $[1,4-7,10,14,16-24]$, and based on clinical relevance and applicability (i.e., available equipment, low administration time, easy to perform tests, and test who are familiar in podiatry care) as judged by the involved podiatrists (D.G., J.B.), physical therapist (S.P.), and human movement scientist (J.B.). Considering the multifactorial causation of falls, the following general fall-risk predictors were considered: fall history in the previous year, fear of falling, feeling unsteady while standing and walking, use of a walking aid, and use of psychoactive medication (NO5A class [antipsychotic drugs], NO5B class [anxiolytic drugs], NO5C class [hypnotic/sedative drugs], NO6A class [antidepressant drugs], NO6B class [psychostimulants and nootropics drugs], N03AE01 [clonazepam], and M03BX07 [tetrazapam]) [20]. In addition, the following foot- and function-related predictors were considered: ankle-dorsiflexion range of motion (ROM), tactile sensitivity of the first metatarsophalangeal joint (1st MPJ), hallux valgus (HV) deformity, lesser toe deformity, foot pain, plantarflexor strength of the hallux, plantarflexor strength of the lesser toes, gait speed, and mediolateral displacement of the center of pressure (CoP) during quiet standing.

The podiatrists performed predictor assessments during history-taking (general fall-risk predictors) and physical examination 
(foot- and function-related predictors) according to a standardized protocol. During history-taking the podiatrist asked the participant whether they had fallen in the previous year, had a fear of falling, felt unsteady while standing and walking, used a walking aid, and used psychoactive medication $[1,10,16,21,23]$. Ankledorsiflexion ROM of both feet was determined by measuring the degrees of the angle between lateral malleolus and fibula with a goniometer, while the participant performed a modified version of the lunge test [25]. This measurement was done for both ankle joints, and the smallest ROM was used in the analysis. Tactile sensitivity of the 1st MPJ was examined using a Semmes-Weinstein 10-g monofilament [5]. Both 1st MPJ were tested when the participants' eyes were closed. The test was considered positive if the participant felt the monofilament $<2$ out of 3 times, on at least one foot [5]. HV deformity was assessed with the 4-point Manchester scale: "no" (0), "mild" (1), "moderate" (2), or "severe" (3) [25]. Scores for both feet were added up (and ranged from 0 to 6). Lesser toe deformities were assessed as per the foot problem score; each lesser toe deformity scored one point [6]. Toe deformity scores of both feet were added up (and ranged from 0 to 16). We assessed foot pain using the Dutch translation of the Foot and Ankle Outcome Score (FAOS)-question: "How often do you experience foot/ ankle pain"? "never" (0), "monthly" (1), "weekly" (2), "daily" (3), or "always" (4). The FAOS is a reliable self-administered questionnaire which is freely available in Dutch [26]. Toe plantarflexor strength was measured with the Paper Grip Test 1 (hallux) and 2 (lesser toes) $[27,28]$. The podiatrist placed a standard business card under the toe(s) and tried to remove the card in a horizontal direction. Both feet were tested, and the test was considered positive when the participant could not grip the card for 3 times or when the test could not be carried out due to a toe amputation on at least one foot [28]. Gait speed was measured as the time in seconds needed to cover a 6-m distance at the participants' usual pace. Participants wore their daily footwear during this test and used a walking aid if needed [19]. The mediolateral displacement of CoP while standing was measured in centimeters with the Footwork Pro Pressure platform software version 5.0.8.1, which is one of the most used platforms in podiatry practices in the Netherlands. Participants stood barefoot for $30 \mathrm{~s}$, with eyes open and heels together with feet toeing out in a $30^{\circ}$ angle [18].

\section{Outcome}

A fall was defined as "an unexpected event in which the participant comes to rest on the ground, floor, or lower level" [29]. Information about the occurrence of falls in a 12-month follow-up was collected by a self-report calendar that the participants received during baseline measurement. At the end of each quarter, the corresponding part of the calendar was returned to the researcher. In addition, each quarter, the participants were contacted by telephone by an evaluator who was blind for baseline data to avoid information bias, as a means of triangulation. If the information on the calendar did not correspond to the information obtained during telephone contact, the participant was called again by an evaluator for clarification. Outcome measurements were assessed by 2 independent researchers (D.G. and J.B.). Doubtful cases were discussed until consensus was reached.

\section{Missing Data}

In case of missing data, the reason for missingness was investigated. To retain a maximum sample size, participants with missing predictor data judged to be missing (completely) at random were measured during the next consultation ( $<9$ weeks from baseline). Participants with missing predictor data for mediolateral displacement of the CoP during analysis were imputed using a last observation carried forward method, based on a recent ( $<6$ months ago from baseline) previous measurement. Participants with unrealistic predictor data during analysis and participants with missing outcome data judged to be missing (completely) at random were removed from analysis using listwise deletion.

\section{Statistical Analysis}

Descriptive statistics were used to summarize participant characteristics. Mann-Whitney $\mathrm{U}$ tests, Fisher exact, and $t$ tests were used to assess differences between fallers and nonfallers.

Before model development, we determined the event per variable (EPV) in our sample, with the aim to maintain an EPV of at least 10 [13]. To this end, we excluded the use of psychoactive medication as a candidate predictor due to insufficient variation $(<10 \%$ or $>90 \%$ present). In addition, collinearity of the remaining predictors was assessed using correlation analyses and calculation of variance inflation factors to identify strongly correlated predictors $(-0.8<$ corr $>+0.8$, variance inflation factor $>10)$ which could be included as one combined predictor [13]. However, no strongly correlated predictors were identified. Furthermore, ordinal predictors were recoded based on clinical relevance and the literature; $\mathrm{HV}$ deformity was recoded into 4 categories (score 0 [0], scores 1 and 2 [1], scores 3 and 4 [2], and scores 5 and 6 [3]), and foot pain was recoded in 3 categories (never [0], monthly and weekly [1], and daily and always [2]). Eventually, our sample had 136 events, and we estimated 16 parameters of 13 variables, resulting in a somewhat lower EPV of 8.5. We have accepted this EPV because the sample size of our study, and thereby, the opportunity to explore the added value of foot-related variables to predict falls is unique for podiatry care.

Model development was performed in 2 steps. In step 1, multivariable logistic regression models were made using groups of predictors in the same category: general fall-risk predictors, foot-related predictors, and function-related predictors. A stepwise backward elimination procedure was applied in each model, removing predictors with a $p$ value $>0.2$. In step 2 , a multivariable logistic regression model was built with the remaining predictors of each group, and a second backward elimination procedure was followed using a $p$ value $>0.2$ as a removal criterion. We chose this $p$ value to retain power to identify predictors with relatively weak effects and to prevent testimation bias, and thus achieve better predictions in new patients [13].

Predictive accuracy of the final model was visualized by the receiver operating characteristic curve and assessed by the area under the curve (AUC). AUCs of 0.5 suggest no discrimination, 0.50.7 were considered poor, $0.7-0.8$ acceptable, $0.8-0.9$ excellent, and $>0.9$ outstanding discrimination. Sensitivity and specificity of the optimal cutoff point (Youden point) were calculated. Fall history in the previous year is a strong, general known predictor and easy to obtain. To determine whether adding foot-related and function-related predictors to this information from history-taking is worth the effort, we compared the AUC of our final model with that of a model that only included fall history in the previous year. DeLong's test was used to test whether the difference between the AUCs was statistically significant. In addition, a reclassification table was used to illustrate how patients change in the pre- 
Fig. 1. Flow diagram of the study popula-

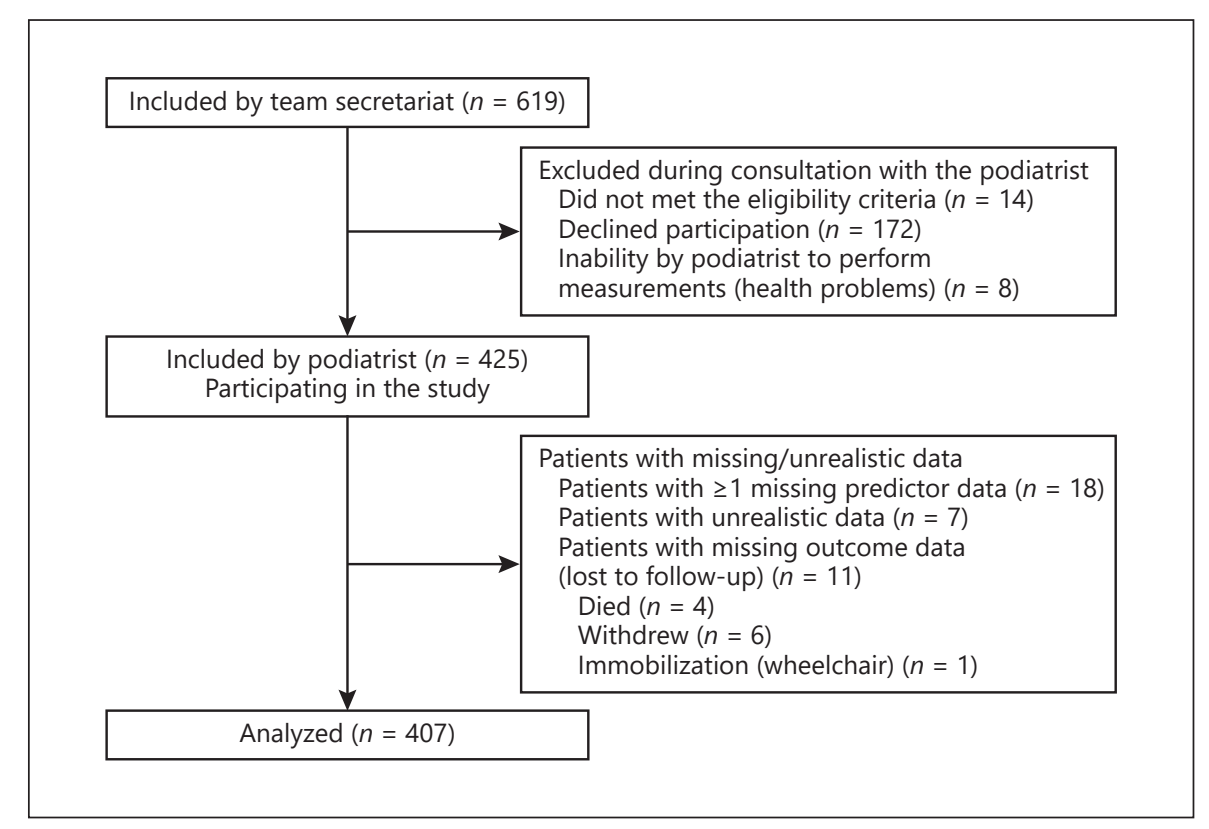
tion.

dicted fall risk category from the model that only include fall history in the previous year compared with our final model using the optimal cutoff point [30]. The performance of the model was also assessed using calibration. A calibration plot was used to visualize the agreement between the outcomes predicted by the model and the actually observed outcomes [13]. The Hosmer-Lemeshow goodness-of-fit test was used to formally test the agreement. A significant result on this test indicates poor agreement. Bootstrap resampling $(n=500)$ was used for internal validation. Bootstrap is a method to estimate the distribution of regression coefficients by resampling with replacement from the original sample [13]. Based on this, a uniform shrinkage factor for the regression coefficients was estimated that should be applied to the original regression coefficients in order to limit the impact of testimation bias [13]. Bootstrap was also used to quantify the optimism in the AUC of the CPM. This optimism was subsequently subtracted from the original estimate to obtain an optimism-corrected AUC. The corrected AUC indicates the expected performance of the model when applied in new patients [13]. A nomogram was plotted to present the final CPM based on the shrunk coefficients. The nomogram can be used to determine the estimated risk of falling for a patient. Statistical analyses were performed using $\mathrm{R}$ version 4.0.3 in Rstudio software for Windows.

\section{Results}

\section{Participants}

During the recruitment period, 619 patients were included, 605 patients met the inclusion criteria, and of these, 425 (70.2\%) consented to participation (Fig. 1). Out of 425,18 participants had missing data which were measured during the next consultation or imputed using the last observation carried forward method. Seven participants had unrealistic data for one predictor, and 11 were lost to follow-up; these participants were excluded from the analysis. Thus, 407 participants remained for analysis. Table 1 presents the participant characteristics. A total of 136 participants experienced at least one fall during follow-up, and 58 of them experienced $>1$ fall. Fallers were significantly older $(p=0.002)$ and had higher prevalence of diabetes $(p=0.003)$ than nonfallers.

\section{Model Development and Specification}

Table 2 presents the results of the multivariable logistic regression analyses for each group of predictors. Table 3 shows the results of the final model. In the final model, 4 predictors were retained: fall history in the previous year, feeling unsteady while standing and walking, plantarflexor strength of the lesser toes, and gait speed.

\section{Model Performance and Internal Validation}

The discrimination of the final CPM was acceptable with an AUC of 0.71 (95\% CI: 0.66\%-0.76\%) (online suppl. Fig. 1; see www.karger.com/doi/10.1159/000520962 for all online suppl. material). The optimal cutoff point in our study sample was at an estimated risk of falling of $30 \%$ (i.e., a risk score below $30 \%$ classifies a patient as nonfaller, and $\geq 30 \%$ as faller), corresponding with a sensitivity of $68 \%$ and specificity of $66 \%$. The discrimination of the model with only fall history in the previous year was statistically significantly $(p<0.001)$ lower, at 0.64 (95\% CI: $0.60-0.69)$. 
Table 1. Patient characteristics (demographics, clinical features, and predictors)

\begin{tabular}{|c|c|c|c|c|}
\hline Characteristic & $\begin{array}{l}\text { Overall } \\
(n=407)\end{array}$ & $\begin{array}{l}\text { Fallers }(n=136) \\
(33.4 \%)\end{array}$ & $\begin{array}{l}\text { Nonfallers } \\
(n=271)(66.6 \%)\end{array}$ & $p$ value ${ }^{b}$ \\
\hline \multicolumn{5}{|l|}{ Demographics and clinical features } \\
\hline Age, mean (SD), years & $74.3(5.8)$ & $75.5(6.0)$ & $73.7(5.6)$ & $0.002^{*}$ \\
\hline Sex, female, $n(\%)$ & $246(60.4)$ & $92(67.6)$ & $154(56.8)$ & $0.041^{*}$ \\
\hline Length, mean (SD), m & $170.8(8.9)$ & $170.3(8.9)$ & $171.1(8.8)$ & 0.406 \\
\hline Weight, mean (SD), kg & $78.6(14.7)$ & $78.5(15.5)$ & $78.6(14.3)$ & 0.958 \\
\hline BMI, mean (SD), $\mathrm{kg} / \mathrm{m}^{2}$ & $26.9(4.6)$ & $27.1(5.0)$ & $26.9(4.4)$ & 0.650 \\
\hline \multicolumn{5}{|l|}{ Medical condition, $n(\%)$} \\
\hline Diabetes & $185(45.5)$ & $76(55.9)$ & $109(40.2)$ & $0.003^{*}$ \\
\hline Osteoarthritis & $219(53.8)$ & $75(55.1)$ & $144(53.1)$ & 0.752 \\
\hline Central nervous system disease & $26(6.4)$ & $12(8.8)$ & $14(5.2)$ & 0.196 \\
\hline \multicolumn{5}{|l|}{ Amputation, $n(\%)$} \\
\hline Hallux & $1(0.2)$ & $0(0.0)$ & $1(0.4)$ & 1.000 \\
\hline \multicolumn{5}{|l|}{ Podiatric intervention, $n$ (\%) } \\
\hline Insoles & $223(54.8)$ & $73(53.7)$ & $150(55.4)$ & 0.752 \\
\hline Insoles + toe orthosis & $14(3.4)$ & $6(4.4)$ & $8(3.0)$ & 0.565 \\
\hline Insoles + brace advice & $1(0.2)$ & $1(0.7)$ & $0(0.0)$ & 0.334 \\
\hline Insoles + tape & $1(0.2)$ & $0(0.0)$ & $1(0.4)$ & 1.000 \\
\hline Toe orthosis & $29(7.1)$ & $7(5.1)$ & $22(8.1)$ & 0.409 \\
\hline Other & $8(2.0)$ & $1(0.7)$ & $7(2.6)$ & 0.278 \\
\hline None & $131(32.2)$ & $48(35.3)$ & $83(30.6)$ & 0.369 \\
\hline \multicolumn{5}{|l|}{ Predictors } \\
\hline \multicolumn{5}{|l|}{ General fall-risk predictors, $n$ (\%) } \\
\hline Fall history in the previous year & $146(35.9)$ & $75(55.1)$ & $71(26.2)$ & $<0.001^{*}$ \\
\hline Fear of falling & $129(31.7)$ & $52(38.2)$ & $77(28.4)$ & 0.055 \\
\hline Unsteady while standing/walking & $183(45.0)$ & $77(56.6)$ & $106(39.1)$ & $0.001^{*}$ \\
\hline Use of a walking aid & $92(22.6)$ & $44(32.4)$ & $48(17.7)$ & $0.001 *$ \\
\hline Use of psychoactive medication & $33(8.1)$ & $14(10.3)$ & $19(7.0)$ & 0.254 \\
\hline \multicolumn{5}{|l|}{ Foot-related predictors } \\
\hline Ankle-dorsiflexion ROM, mean (SD), degree & $20.5(7.1)$ & $19.7(6.5)$ & $20.9(7.4)$ & 0.099 \\
\hline Tactile sensitivity 1 st MPJ, $n(\%)$ & $125(30.7)$ & $55(40.4)$ & $70(25.8)$ & $0.003^{*}$ \\
\hline \multicolumn{5}{|l|}{ Toe deformity } \\
\hline HV deformity ${ }^{\mathrm{a}}$, median (IQR) & $2(0-3)$ & $2(0-3)$ & $2(0-3)$ & 0.293 \\
\hline Lesser toe deformity, median (IQR) & $2(0-5)$ & $2(0-6)$ & $2(0-4)$ & 0.080 \\
\hline Foot pain, median (IQR) & $2(0-3)$ & $1(0-3)$ & $2(0-3)$ & 0.987 \\
\hline \multicolumn{5}{|l|}{ Toe plantarflexor strength } \\
\hline Hallux, $n$ (\%) (fail) & $110(27.0)$ & $46(33.8)$ & $64(23.6)$ & $0.033^{*}$ \\
\hline Lesser toes, $n$ (\%) (fail) & $129(31.7)$ & $55(40.4)$ & $74(27.3)$ & $0.009^{*}$ \\
\hline \multicolumn{5}{|l|}{ Function-related predictors } \\
\hline Gait speed, median (IQR), s & $5.7(4.6-7.0)$ & $6.1(5.1-7.6)$ & $5.5(4.5-6.5)$ & $<0.001^{*}$ \\
\hline ML displacement CoP, median (IQR), cm & $1.6(1.1-2.3)$ & $1.7(1.2-2.4)$ & $1.6(1.1-2.2)$ & 0.152 \\
\hline
\end{tabular}

The reclassification table (online suppl. Table 1) shows that our model assigned more fallers correctly to a higher predicted risk category (26\%) than nonfallers incorrectly to a higher predicted risk category (11\%). Bootstrap resampling yielded shrinkage factors of -0.1713 (intercept) and 0.7283 (coefficients). The optimism-corrected AUC was 0.65.
Figure 2 shows the calibration plot of the final CPM. The Hosmer-Lemeshow goodness-of-fit test was not significant $(p=0.892)$. Online supplementary Figure 2 presents the nomogram of the final CPM, based on the shrunk beta-coefficients. 
Table 2. Results of multivariable logistic regression analysis per subcategory for falls

Odds ratio $p$ value

\begin{tabular}{|c|c|c|}
\hline \multicolumn{3}{|l|}{$\begin{array}{l}\text { Group: general fall-risk predictors } \\
\text { Full block }\end{array}$} \\
\hline Fall history in the previous year & 3.21 & $<0.001$ \\
\hline Fear of falling & 1.25 & 0.377 \\
\hline Unsteady while standing/walking & 1.51 & 0.102 \\
\hline Use of a walking aid & 1.42 & 0.208 \\
\hline \multicolumn{3}{|l|}{ Retained predictors } \\
\hline Fall history in the previous year & 3.16 & $<0.001$ \\
\hline Unsteady while standing/walking & 1.60 & 0.052 \\
\hline Use of a walking aid & 1.49 & 0.148 \\
\hline \multicolumn{3}{|l|}{ Group: foot-related predictors } \\
\hline \multicolumn{3}{|l|}{ Full block } \\
\hline Ankle-dorsiflexion ROM & 0.98 & 0.206 \\
\hline Tactile sensitivity 1st MPJ & 1.77 & 0.014 \\
\hline \multicolumn{3}{|l|}{ Toe deformity } \\
\hline \multicolumn{3}{|l|}{ HV deformity } \\
\hline HV 0 (reference) & - & \\
\hline HV 1 & 0.97 & 0.916 \\
\hline HV 2 & 1.12 & 0.727 \\
\hline HV 3 & 1.13 & 0.781 \\
\hline Lesser toe deformity & 1.03 & 0.312 \\
\hline \multicolumn{3}{|l|}{ Foot pain } \\
\hline Foot pain 0 (reference) & - & \\
\hline Foot pain 1 & 1.21 & 0.486 \\
\hline Foot pain 2 & 0.95 & 0.830 \\
\hline \multicolumn{3}{|l|}{ Toe plantarflexor strength } \\
\hline Hallux & 1.23 & 0.458 \\
\hline Lesser toes & 1.45 & 0.156 \\
\hline \multicolumn{3}{|l|}{ Retained predictors } \\
\hline Tactile sensitivity 1 st MPJ & 1.78 & 0.012 \\
\hline Toe plantarflexor strength - lesser toes & 1.62 & 0.033 \\
\hline \multicolumn{3}{|l|}{$\begin{array}{l}\text { Group: function-related predictors } \\
\text { Full block }\end{array}$} \\
\hline Gait speed & 1.14 & 0.001 \\
\hline ML displacement CoP & 1.12 & 0.255 \\
\hline \multicolumn{3}{|l|}{ Retained predictors } \\
\hline Gait speed & 1.15 & $<0.001$ \\
\hline
\end{tabular}

$\mathrm{ML}$, mediolateral.

\section{Discussion}

The aim of this study was to develop and internally validate a CPM to predict falls in the podiatry community-dwelling older adult population. The final CPM includes fall history in the previous year, feeling unsteady while standing and walking, decreased plantarflexor strength of the lesser toes, and reduced gait speed. This model achieved acceptable accuracy and outperformed a prediction based on fall history alone. However, the accuracy of the model in clinical practice should be confirmed in future validation studies. Predictors for falls found in this study were consistent to those found in previous studies building a CPM [14, 16, 17, 20, 21, 23, 24]. Fall history in the previous year and feeling unsteady while standing and walking have frequently been identified as predictors for falls and included in various CPMs, as well as in an algorithm based on the American Geriatrics Society and British Geriatrics Society guideline [16, $17,20,21,23,24]$. In addition, as in the study by Menz et al. [14], we identified decreased plantarflexor strength of the lesser toes as a significant predictor. A decline in toe plantarflexor strength is associated with older age [27]. Considering the important role of the toes in maintaining balance during standing and walking, it is understandable why loss of toe plantarflexor strength in older adults leads to increased risk of falls [14]. The predictive value of gait speed has also been previously identified [10, 17]. Gait abnormalities due to pain, muscle weakness, joint immobility, rigidity, spasticity and poor functioning of the central nervous system, as well as sensory deficits in the lower extremities can express in slower gait speed, and result in an increased risk of falling $[17,31]$.

In contrast with findings of Menz et al. [14], foot pain was not retained in our final model. This contradictory result in our study is most likely due to the podiatric intervention(s) performed at baseline (for more specification, see Table 1) or during follow-up. In case of an effective intervention, foot pain will have reduced during the follow-up period, thereby no longer contributing to the risk of falls [32]. A priori, we expected a lower percentage of foot pain within fallers than that with the current literature due to the effect of the podiatric interventions in our sample. However, since foot pain was significantly associated with falls in several previous studies [4, $7,14]$, and recommended to be included in a fall risk assessment in a systematic review [7], we included foot pain as candidate predictor nonetheless. In addition, not all participants receive interventions aimed at pain reduction, and foot pain, such as chronic or neuropathic pain, can not always be reduced. Obviously, we could not withhold participants from interventions due to ethical considerations, and this would also have resulted in a nonrepresentative sample for clinical practice. Another explanation for the lack of association of falling with foot pain might be that we used a different scale to assess foot pain, compared to previous studies. We used a single question from the FAOS to measure foot pain, while, for example, the Manchester Foot Pain and Disability Index 
Table 3. Multivariable logistic regression analysis for falls: final model

\begin{tabular}{lllll}
\hline & $\begin{array}{l}\text { Regression coefficient } \\
\text { (intercept =-2.021) }\end{array}$ & $\begin{array}{l}\text { Odds ratio } \\
(95 \% \mathrm{Cl})\end{array}$ & $\begin{array}{c}p \text { value } \\
\text { Shrunk regression } \\
\text { coefficient } \\
\text { (intercept =-2.192) }\end{array}$ & $\begin{array}{l}\text { Shrunk } \\
\text { odds ratio }\end{array}$ \\
\hline $\begin{array}{l}\text { General fall-risk predictors } \\
\text { Fall history in the previous year } \\
\quad \text { Unsteady while standing/walking }\end{array}$ & 1.133 & $3.10(2.00-4.86)$ & $<0.001$ & 0.825 \\
$\begin{array}{l}\text { Foot-related predictors } \\
\text { Toe plantarflexor strength } \\
\quad \text { Lesser toes }\end{array}$ & 0.434 & $1.54(0.98-2.44)$ & 0.063 & 0.316 \\
$\begin{array}{l}\text { Function-related predictors } \\
\text { Gait speed }\end{array}$ & 0.341 & $1.41(0.88-2.25)$ & 0.155 & 0.248 \\
\hline Cl, confidence interval. & 0.087 & $1.09(1.01-1.18)$ & 0.033 & 0.063 \\
\hline
\end{tabular}

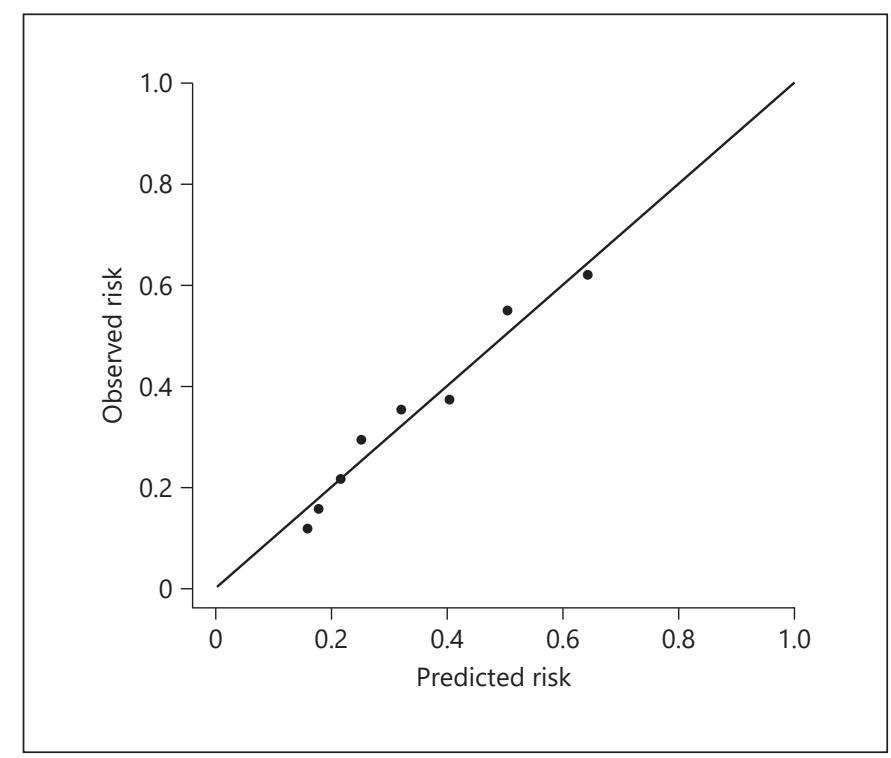

Fig. 2. Calibration plot of the final CPM which showed the predicted risk versus the observed risk in the dataset. There are 8 risk score strata defined, each containing patients $(>50)$ with similar predicted probabilities of the outcome event. The $45^{\circ}$ line reflects the maximum agreement between observed and expected risk (perfect calibration) [13].

and the Visual Analog Scale were used in previous studies. We did consider these other scales during the design of the study, but had judged them unsuitable because they showed high failure rates in older adults [33], measured only chronic pain [7], were not described in detail, or were not (freely) available in Dutch, which would hinder the implementation of the CPM.
In addition, the use of psychoactive medication, included as an important predictor in previous models and initially under consideration for the model in our study, could not be investigated because of the low percentage of use $(<10 \%)$. The percentage of psychoactive medication use in this study $(8.1 \%)$ is lower than that described in previous studies $(17-38 \%)$ [20, 22]. An explanation could be the current relative reticence of prescribing psychoactive medication in the Netherlands because of the associated serious side effects [34]. It should be noted that the low percentage could also be the result of the ignorance of the use by the participant, despite the explanation by the podiatrist about the type of medication. Regardless, based on the current study, we can not make any statements on its current association with fall risk.

Performance of our model is relatively good in comparison with existing tools which can be used in clinical (podiatry) practice to predict the occurrence of any fall in community-dwelling older adults. AUCs of existing models were between 0.51 and 0.70 [1, 20, 24, 35-40]. Only one case-control study of Kim and Xiong [41] shows an AUC of 0.85 for the shortened version of the Falls Efficacy Scale (FES-I) and an AUC of 0.75 for the Berg Balance Scale (BBS). However, other studies show limited predictive value for both the FES-I and BBS [10, 42]. The accuracy as reported in the study of Kim and Xiong [41] is likely overestimated due to the case-control study design. In addition, a prospective study of Hirase et al. [21] shows an AUC of 0.73 and of Okochi et al. [23] shows an AUC of 0.74 ; however, they used a follow-up period of 3 months and 6 months instead of 12 months. Furthermore, existing tools are currently scarcely implemented in clinical practice [12]. We expect easier implementation 
of our model in clinical podiatry care because the CPM is specifically developed for the podiatry community-dwelling older adult population. Our model consists of familiar and clinical applicable tests for the podiatrist, and the administration time is short $(<5 \mathrm{~min})$. Although a predictive association does not by definition imply causality, the items included in the CPM could also support podiatrists in their actions to prevent falls. For example, if a patient is deemed at risk because of positive scores on feeling unsteady while standing and walking and/or having a slow walking speed, the underlying reasons can be further investigated, and appropriate podiatric action can be taken. Such reasons may include pain, insufficient support by the feet, inappropriate footwear, deficits in somatosensory feedback, or insufficient foot muscle strength. Accordingly, appropriate (multifaceted) treatment may include footwear advice, orthoses, and foot and ankle exercises, or referral to a relevant specialist or fall prevention program [15].

Some limitations to our study should be mentioned. First, recall bias cannot completely be eliminated in this older adult population. We tried to limit this by contacting the participants by telephone in addition to the calendar. We also tried to avoid recall bias with a prospective design; therefore, we expect it has had limited effect on our results. Second, despite our aim to keep the EPV ratio $\geq 10$ :1, we ended up with a somewhat less favorable ratio. Although accepting this ratio enabled us to explore the foot-related predictors more elaborately, the trade-off is that our model is likely slightly overfit $(\mathrm{EPV}<10)[13]$. To improve the quality of predictions for future patients, we have shrunk the estimated regression coefficients to less extreme values, using Bootstrap resampling. Third, all predictors were assessed by multiple podiatrists in clinical practice. Although podiatrists were trained by instruction videos and performed predictor assessment according to a written protocol, some random error in measurements cannot be excluded. On the other hand, this does reflect the way these variables are collected in clinical practice. Fourth, in order to maintain the clinical applicability of the model, we performed only a single measurement of $30 \mathrm{~s}$ to obtain the mediolateral displacement of the CoP. This avoids the problem of the measurement being too time consuming in clinical practice, but it could have compromised the reliability of the measurement. In addition, the Paper Grip Test is used to measure foot muscle strength because it is a simple and clinically applicable test. However, the rate of force applied by the examiner could probably influence the outcome of the test [28]. We tried to eliminate variability as much as pos- sible by standardizing the type of paper and underground used in our study, but it is not possible to standardize the force applied by the examiner to fully eliminate this effect. Furthermore, when used in clinical practice, the podiatrists needs to pay attention that the heel is on the ground during the Paper Grip Test to prevent the contribution of the ankle plantarflexors as much as possible [27]. Finally, not all possible risk factors could be included as candidate predictors in the study due to the risk of overfitting of the model. So, a priori, we have only selected the most strong and clinically applicable predictors in podiatry practice as candidate predictors. Thus, demographic and clinical factors such as age and diabetes were not considered as candidate predictors because they have not been unambiguously identified as significant predictors among community-dwelling older adults in previous research $[1,10,42]$. Nevertheless, due to the significant association between age and diabetes with falls in our sample (for more specification see Table 1), we performed a post hoc analysis to test the added value of diabetes and age to the current model. This analysis showed no improvement in the Akaike information criterion, a method to evaluate the model fit on the data [13], and the AUC of the model. In addition, footwear assessment was also not included as a candidate predictor, despite the fact that previous studies showed that inappropriate footwear can contribute to falls $[1,3,9]$. The use of footwear as a predictor is problematic because most people do not wear the same shoes every day or even walk barefoot indoors. The study also had some notable strengths; we included a wide range of foot-related variables, in addition to general fall-risk and function-related factors, and we developed the model in a specified target population; the podiatry communitydwelling older adult population, which is unique in this research field.

\section{Conclusion}

In conclusion, our CPM based on fall history in the previous year, feeling unsteady while standing and walking, decreased plantarflexor strength of the lesser toes, and reduced gait speed has acceptable accuracy to predict falls in our sample of podiatry community-dwelling older adults and is easily applicable in this setting. The accuracy of the model in clinical practice should be demonstrated through external validation of the model in a next study. 


\section{Acknowledgments}

The authors gratefully acknowledge the secretariat and podiatrists of RondOm Podotherapeuten, Podiatric Primary Care Center, Leusden, for the recruitment of patients and data collection for the present study.

\section{Statement of Ethics}

This study was waived from formal medical ethical evaluation by the Dutch Central Committee on Research Involving Human Subject (CCMO). Ethical standards adhered to the Declaration of Helsinki. All patients gave written informed consent.

\section{Conflict of Interest Statement}

The authors declared that they have no competing interests.

\section{Funding Sources}

This work was funded (50\%) by the Dutch association of podiatrists (NVvP: Nederlandse Vereniging van Podotherapeuten). The funder had no influence on study design, recruitment, data collection, analysis, and writing of the article.

\section{Author Contributions}

D.J.J.G., J.G.M.B., and S.I.B.P. conceived and designed the study. The secretariat of RondOm recruited participants and the podiatrist of RondOm collected data. D.J.J.G. and M.M.S. conducted the statistical analysis. D.J.J.G., M.M.S., M.L., and C.L. interpretated the data. D.J.J.G. drafted the manuscript. All authors critically revised the draft manuscript and agreed to the final version.

\section{Data Availability Statement}

The data that support the findings of this study are not publicly available due to their containing information that could compromise the privacy of research participants but are available from the corresponding author (D.J.J.G.) upon reasonable request.

\section{References}

1 Federation of Medical Specialists. Prevention of falls in the older adult. Guideline 2017 [Internet]. (Dutch: original tile: Federatie Medisch Specialisten. Preventie van valincidenten bij ouderen. Richtlijn 2017) [cited 2018 May 31]. Available from: https://richtlijnendatabase.nl/richtlijn/preventie_van_valincidenten_bij_ouderen/startpagina_-_preventie_van_valincidenten.html.

2 VeiligheidNL, Center of Expertise Injury Prevention. Fallprevention: facts and figures (Dutch: original title: Veiligheid NL, Cijfers Valongevallen 65+ in 2019) [Internet]. [cited 2020 Dec 10]. Available from: https://www. veiligheid.nl/valpreventie/feiten-cijfers.

3 Panel on Prevention of Falls in Older Persons American Geriatrics Society and British Geriatrics Society. Summary of the Updated American Geriatrics Society/British Geriatrics Society clinical practice guideline for prevention of falls in older persons. J Am Geriatr Soc. 2011;59(1):148-57.

4 Menz HB, Auhl M, Spink MJ. Foot problems as a risk factor for falls in community-dwelling older people: a systematic review and meta-analysis. Maturitas. 2018;118:7-14.

5 Carrer P, Trevisan C, Curreri C, Giantin V, Maggi S, Crepaldi G, et al. Semmes-Weinstein monofilament examination for predicting physical performance and the risk of falls in older people: results of the Pro.V.A. Longitudinal Study. Arch Phys Med Rehabil. 2018; 99(1):137-43.e1

6 Menz HB, Lord SR. The contribution of foot problems to mobility impairment and falls in community-dwelling older people. J Am Geriatr Soc. 2001;49(12):1651-6.

7 Stubbs B, Binnekade T, Eggermont L, Sepehry AA, Patchay S, Schofield P. Pain and the risk for falls in community-dwelling older adults: systematic review and meta-analysis. Arch Phys Med Rehabil. 2014;95(1):175-87.e9.

8 Vernon W, Borthwick A, Walker J. The management of foot problems in the older person through podiatry services. Rev Clin Gerontol. 2011;21(4):331-9.

9 National Institute for Health and Care Excellence-NICE. Falls in older people: assessing risk and prevention [Internet]. London: NICE; 2013 [cited 2021 Jun 6]. Available from: https://www.nice.org.uk/guidance/ cg161/chapter/Introduction.

10 Lusardi MM, Fritz S, Middleton A, Allison L, Wingood M, Phillips E, et al. Determining risk of falls in community dwelling older adults: a systematic review and meta-analysis using Posttest probability. J Geriatr Phys Ther. 2017;40(1):1-36.

11 Park SH. Tools for assessing fall risk in the older adult: a systematic review and metaanalysis. Aging Clin Exp Res. 2018 Jan;30(1): $1-16$.

12 van Rhyn B, Barwick A. Health practitioners' perceptions of falls and fall prevention in older people: a metasynthesis. Qual Health Res. 2019;29(1):69-79.

13 Steyerberg EW. Clinical prediction models: a practical approach to development, validation, and updating. 2nd ed. Cham, Switzerland: Springer; 2019.
14 Menz HB, Morris ME, Lord SR. Foot and an kle risk factors for falls in older people: a prospective study. J Gerontol A Biol Sci Med Sci. 2006;61(8):866-70.

15 Wylie G, Torrens C, Campbell P, Frost H, Gordon AL, Menz HB, et al. Podiatry interventions to prevent falls in older people: a systematic review and meta-analysis. Age Ageing. 2019;48(3):327-36.

16 Stevens JA, Phelan EA. Development of STEADI: a fall prevention resource for health care providers. Health Promot Pract. 2013; 14(5):706-14.

17 Luukinen H, Koski K, Laippala P, Kivelä SL. Predictors for recurrent falls among the home-dwelling elderly. Scand J Prim Health Care. 1995;13(4):294-9.

18 Piirtola M, Era P. Force platform measurements as predictors of falls among older people: a review. Gerontology. 2006;52(1):1-16.

19 Tiedemann A, Shimada H, Sherrington C, Murray S, Lord S. The comparative ability of eight functional mobility tests for predicting falls in community-dwelling older people. Age Ageing. 2008;37(4):430-5.

20 Bongue $\mathrm{B}$, Dupré $\mathrm{C}$, Beauchet $\mathrm{O}$, Rossat $\mathrm{A}$, Fantino B, Colvez A. A screening tool with five risk factors was developed for fall-risk prediction in community-dwelling elderly. J Clin Epidemiol. 2011;64(10):1152-60.

21 Hirase T, Inokuchi S, Matsusaka N, Nakahara K, Okita M. A modified fall risk assessment tool that is specific to physical function predicts falls in community-dwelling elderly people. J Geriatr Phys Ther. 2014;37(4):159-65. 
22 Landi F, Onder G, Cesari M, Barillaro C, Russo A, Bernabei R, et al. Psychotropic medications and risk for falls among communitydwelling frail older people: an observational study. J Gerontol A Biol Sci Med Sci. 2005; 60(5):622-6.

23 Okochi J, Toba K, Takahashi T, Matsubayashi K, Nishinaga M, Takahashi R, et al. Simple screening test for risk of falls in the elderly. Geriatr Gerontol Int. 2006;6(4):223-7.

24 Tromp AM, Pluijm SM, Smit JH, Deeg DJ, Bouter LM, Lips P. Fall-risk screening test: a prospective study on predictors for falls in community-dwelling older adult. J Clin Epidemiol. 2001;54(8):837-44.

25 Menz HB, Tiedemann A, Kwan MM, Latt MD, Sherrington C, Lord SR. Reliability of clinical tests of foot and ankle characteristics in older people. J Am Podiatr Med Assoc. 2003;93(5):380-7.

26 Sierevelt IN, Beimers L, van Bergen CJA, Haverkamp D, Terwee CB, Kerkhoffs GMMJ. Validation of the Dutch language version of the foot and ankle outcome score. Knee Surg Sports Traumatol Arthrosc. 2015;23(8): 2413-9.

27 Menz HB, Zammit GV, Munteanu SE, Scott G. Plantarflexion strength of the toes: age and gender differences and evaluation of a clinical screening test. Foot Ankle Int. 2006;27(12): 1103-8.

28 de Win MM, Theuvenet WJ, Roche PW, de Bie RA, van Mameren $H$. The paper grip test for screening on intrinsic muscle paralysis in the foot of leprosy patients. Int J Lepr Other Mycobact Dis. 2002;70(1):16-24.
29 Lamb SE, Jørstad-Stein EC, Hauer K, Becker C; Prevention of Falls Network Europe and Outcomes Consensus Group. Development of a common outcome data set for fall injury prevention trials: the Prevention of Falls Network Europe consensus. J Am Geriatr Soc. 2005;53(9):1618-22.

30 Wijeysundera DN. Predicting outcomes: is there utility in risk scores? Can J Anaesth. 2016;63(2):148-58.

31 Trueblood PR, Rubenstein LZ. Assessment of instability and gait in elderly persons. Compr Ther. 1991;17(8):20-9.

32 Menz HB. Chronic foot pain in older people. Maturitas. 2016;91:110-4.

33 Bergh I, Sjöström B, Odén A, Steen B. An application of pain rating scales in geriatric patients. Aging. 2000;12(5):380-7.

34 Foundation for Pharmaceutical key figures. Use of benzodiazepines decreased further last year [Internet]. (Dutch: original tilte: Stichting Farmaceutische Kerngetallen. Gebruik benzodiazepines vorig jaar verder afgenomen) [cited 2018 May 10]. Available from: https:// www.sfk.nl/publicaties/PW/2018/gebruikbenzodiazepines-vorig-jaar-verder-afgenomen.

35 Russell MA, Hill KD, Blackberry I, Day LM, Dharmage SC. The reliability and predictive accuracy of the falls risk for older people in the community assessment (FROP-Com) tool. Age Ageing. 2008;37(6):634-9.

36 Palumbo P, Palmerini L, Bandinelli S, Chiari L. Fall risk assessment tools for older adult living in the community: can we do better? PLoS One. 2015;10(12):e0146247.
37 Lohman MC, Crow RS, DiMilia PR, Nicklett EJ, Bruce ML, Batsis JA. Operationalisation and validation of the Stopping Older adult Accidents, Deaths, and Injuries (STEADI) fall risk algorithm in a nationally representative sample. J Epidemiol Community Health. 2017;71(12):1191-7.

38 Mielenz TJ, Kannoth S, Jia H, Pullyblank K, Sorensen J, Estabrooks P, et al. Evaluating a two-level vs. three-level fall risk screening algorithm for predicting falls among older adults. Front Public Health. 2020;8:373.

39 Chen PL, Lin HY, Ong JR, Ma HP. Development of a fall-risk assessment profile for community-dwelling older adults by using the National Health Interview Survey in Taiwan. BMC Public Health. 2020;20:234.

40 Balasubramanian CK, Boyette A, Wludyka P. How well do functional assessments of mobility and balance discriminate fallers and recurrent fallers from non-fallers among ambulatory older adults in the community? Physiother Can. 2015;67(2):184-93.

41 Kim T, Xiong S. Comparison of seven fall risk assessment tools in community-dwelling Korean older women. Ergonomics. 2017;60(3): 421-9.

42 Kamide N, Shiba Y, Sakamoto M, Sato H, Kawamura A. Fall-related efficacy is a useful and independent index to detect fall risk in Japanese community-dwelling older people: a 1-year longitudinal study. BMC Geriatr. 2019; 19(1):293. 